\title{
CONSTITUINTES QUÍMICOS E ATIVIDADE INSETICIDA DOS EXTRATOS DE FRUTOS DE Trichilia elegans E T. catigua (MELIACEAE)
}

\author{
Andréia Pereira Matos, Liliane Nebo, Paulo Cezar Vieira*, João Batista Fernandes e Maria Fátima das Graças Fernandes \\ da Silva \\ Departamento de Química, Universidade Federal de São Carlos, CP 676, 13560-970 São Carlos - SP, Brasil \\ Ricardo Ribeiro Rodrigues \\ Departamento de Ciências Biológicas, Escola Superior de Agricultura “Luiz de Queiroz", Universidade de São Paulo, CP 9, \\ 13418-900 Piracicaba - SP, Brasil
}

Recebido em 18/9/08; aceito em 16/2/09; publicado na web em 3/7/09

\begin{abstract}
CHEMICAL CONSTITUENTS AND INSECTICIDAL ACTIVITY FROM FRUITS EXTRACTS OF Trichilia elegans AND $T$. catigua (Meliaceae). Phytochemical investigation of the fruits extracts of Trichilia elegans and Trichilia catigua (Meliaceae) has led to the identification of the limonoids $11 \beta$-acetoxyobacunone, cedrelone, methylangolensate and epimeric mixture of photogedunin besides known coumarins (scoparone, scopoletin, umbeliferone) and the steroids stigmasterol, $\beta$-sitosterol, sitostenone and campesterol. The structures of the compounds were proposed by spectroscopic analysis and comparison with literature data. An evaluation of the insecticidal activity of the fruits extracts of Trichilia ssp. was carried out and the extracts of T. elegans revealed to have strong insecticidal activity and the extracts of T. catigua showed moderate larval mortality on Spodoptera frugiperda.
\end{abstract}

Keywords: Trichilia; limonoids; Spodoptera frugiperda.

\section{INTRODUÇÃO}

O uso de plantas com propriedades inseticidas tem se mostrado uma promissora ferramenta no controle de insetos, por causar menor impacto ambiental, o que atende aos anseios da sociedade moderna. ${ }^{1}$ Dentre as espécies utilizadas como inseticida, o nim, Azadirachta indica (Meliaceae), é a popularmente mais conhecida e cujo espectro de ação é bastante amplo, tendo seu efeito comprovado sobre aproximadamente 400 espécies de insetos. ${ }^{2}$ A partir dos excelentes resultados verificados com o nim, outras meliáceas também passaram a despertar o interesse de pesquisadores, no intuito de encontrar novas espécies e novas moléculas com atividade inseticida. Várias espécies de Meliaceae se destacam entre as famílias botânicas que apresentam ingredientes ativos com atividade inseticida, proporcionando eficiência de seus extratos. ${ }^{3} \mathrm{O}$ gênero Trichilia é um deles, pois além de apresentar compostos com atividade inseticida conhecida (triquilinas), ${ }^{4}$ é bastante abundante nas regiões tropicais da América com mais de 230 espécies conhecidas. ${ }^{5}$

Mikolajczak e Reed $^{6}$ realizaram um dos primeiros trabalhos testando a atividade inseticida de Trichilia sobre S. frugiperda. Os extratos etanólicos de T. pallida, T. connoroides, T. prieureana, $T$. roka e $T$. triphyllaria testados causaram mortalidade igual ou superior a $80 \%$ das lagartas, sendo que apenas a última espécie não afetou a sobrevivência do inseto. Diversos trabalhos comprovando a atividade inseticida de Trichilia ssp. sobre Spodoptera frugiperda têm sido realizados. ${ }^{17,8} \mathrm{~A}$ atividade biológica sobre insetos de alguns limonoides isolados de espécies de Trichilia vem sendo investigada, e resultados promissores foram encontrados. ${ }^{4,9-11}$

A espécie Trichilia elegans ssp. elegans A. Juss, conhecida popularmente como "Cachuá", produz madeira resistente e durável, ocorre em matas semidecíduas e possui ampla dispersão no país, sendo encontrada desde Goiás até Santa Catarina, apresenta-se como uma árvore de 3 a 6 m de altura. ${ }^{12}$ Já a espécie Trichilia catigua A. Juss, são árvores com cerca de $10 \mathrm{~m}$ de altura e possuem flores de

*e-mail: paulo@dq.ufscar.br coloração branco-amareladas. Popularmente, são conhecidas como catiguá, caatiguá, cedrinho e angelim-rosa, e têm ocorrência de São Paulo até o Rio Grande do Sul. Recentemente, ensaios biológicos realizados sobre $S$. frugiperda com os extratos dos ramos, folhas e frutos de T. elegans e T. catigua evidenciaram efeitos significativos sobre este inseto, o que serviu de estímulo para o estudo químico destas espécies.

O presente trabalho descreve o isolamento e a caracterização estrutural das cumarinas escoporona (1), escopoletina (2), umbeliferona (3), o limonoide $11 \beta$-acetoxiobacunona (4), e a mistura dos esteroides estigmasterol, $\beta$-sitosterol, sitostenona e campesterol para a espécie T. elegans, e os limonoides cedrelona (5), angolensato de metila (6) e mistura epimérica de fotogedunina (7 e 8) para a espécie $T$. catigua. A presença de limonoides em sementes de T. elegans já havia sido anteriormente registrada, ${ }^{13}$ porém o limonoide $\mathbf{4}$ está sendo relatado pela primeira vez no gênero Trichilia; já as demais substâncias estão sendo descritas pela primeira vez em suas respectivas espécies. Encontra-se também neste trabalho o primeiro relato do isolamento de limonoides na espécie T. catigua.

\section{PARTE EXPERIMENTAL}

\section{Procedimentos experimentais gerais}

Os espectros de RMN ${ }^{1} \mathrm{H}$ e ${ }^{13} \mathrm{C}$ (uni e bidimensionais) foram obtidos em espectrômetro Bruker DRX $400 \mathrm{MHz}$, utilizando-se $\mathrm{CDCl}_{3}$ e $\mathrm{CD}_{3} \mathrm{OD}$ como solventes e TMS como padrão interno. Os espectros de massas forma obtidos em aparelho da Micromass, modelo Micromass Quattro LC.

Para identificação dos esteroides foi utilizado cromatógrafo com fase gasosa acoplado ao espectrômetro de massas, marca Shimadzu, modelo QP-5000, com coluna capilar DB-5 (30 m x 0,25 mm), utilizando as seguintes condições: temperatura do injetor: $250{ }^{\circ} \mathrm{C}$, gás de arraste: $\mathrm{He}$, temperatura inicial do forno: $150{ }^{\circ} \mathrm{C}$ por $1 \mathrm{~min}$, velocidade de aquecimento a $6{ }^{\circ} \mathrm{C} / \mathrm{min}$ até $280{ }^{\circ} \mathrm{C}$, permanecendo nessa temperatura por $15 \mathrm{~min}$. $\mathrm{O}$ espectro de massas foi obtido por impacto de elétrons a $70 \mathrm{eV}$. 
As separações cromatográficas em colunas foram realizadas utilizando-se gel de sílica 60, 70-230, 230-400 mesh e Sephadex LH20. As separações por cromatografia líquida de alta eficiência (CLAE) em condições preparativas foram realizadas em coluna Asahipak GS-310 2G (2,15 x 50,0 cm), utilizando equipamento Shimadzu LC$8 \mathrm{~A}$ com válvula de reciclo, injetor Rheodyne 7123 com loop $500 \mu \mathrm{L}$ e detector UV/VIS Shimadzu SPD-6AV.

As análises cromatográficas em camada fina foram realizadas em cromatoplacas de sílica gel $\mathrm{F}_{254}$ sobre placa de alumínio Merck, de $0,2 \mathrm{~mm}$ de espessura, empregando-se como revelador a solução de vanilina/ácido sulfúrico.

\section{Material vegetal}

Os frutos de T. elegans e T. catigua foram coletados em Piracicaba/SP no Campus da Escola Superior de Agricultura "Luiz de Queiroz" (Esalq/USP) e identificados pelo botânico Dr. Ricardo Ribeiro Rodriguez. Uma exsicata de T. elegans e outra de T. catigua foram depositadas sob os números 80407 e 7394, respectivamente.

\section{Preparação dos extratos}

Os frutos secos $(84,0 \mathrm{~g})$ e moídos de T. elegans foram extraídos à temperatura ambiente e em repouso, com solventes em ordem crescente de polaridade (hexano, $\mathrm{MeOH}$ e MeOH/água 1:1). As soluções obtidas foram concentradas por destilação do solvente em evaporador rotativo, fornecendo extratos hexânico, metanólico e hidrometanólico. Os frutos de T. catigua foram separados em arilo $(3,5 \mathrm{~g})$, semente $(8,0 \mathrm{~g})$ e exocarpo $(3,0 \mathrm{~g})$ e, posteriormente, secos e moídos. A extração das partes dos frutos de T. catigua foi realizada utilizando as mesmas condições descritas para os frutos de $T$. elegans. Os extratos foram submetidos a ensaios biológicos sobre a lagarta-do-cartucho $S$. frugiperda e os que apresentaram atividade foram fracionados.

\section{Isolamento das substâncias de T. elegans}

O extrato hexânico dos frutos de T. elegans $(4,20 \mathrm{~g})$ foi submetido à cromatografia em coluna líquida a vácuo empacotada com sílica gel (230-400 mesh), utilizando-se como fases móveis solventes orgânicos de polaridade crescente: hexano; hexano/AcOEt 9:1; AcOEt e MeOH. Foram obtidas 4 frações após evaporação dos solventes orgânicos: $\mathrm{FH} 1=20 \mathrm{mg} ; \mathrm{FH} 2=2800 \mathrm{mg} ; \mathrm{FH} 3=970 \mathrm{mg}$ e FH4 = $245 \mathrm{mg}$. A fração FH3 foi submetida a vários fracionamentos em coluna de sílica gel (70-230 mesh), eluída com $\mathrm{CH}_{2} \mathrm{Cl}_{2} / \mathrm{MeOH}\left(\mathrm{CH}_{2} \mathrm{Cl}_{2} ; \mathrm{CH}_{2} \mathrm{Cl}_{2} /\right.$ $\mathrm{MeOH}(1,0 \rightarrow 50,0 \%) ; \mathrm{MeOH})$, em gradiente de polaridade crescente fornecendo as substâncias $\mathbf{1}(1,0 \mathrm{mg}), \mathbf{2}(1,0 \mathrm{mg}), \mathbf{3}(1,6 \mathrm{mg})$ e a mistura dos esteroides $(8,0 \mathrm{mg})$.

O extrato metanólico dos frutos de T. elegans $(5,20 \mathrm{~g})$ foi suspenso em uma mistura $\mathrm{MeOH} / \mathrm{H}_{2} \mathrm{O}$ e particionado sucessivamente com $\mathrm{CH}_{2} \mathrm{Cl}_{2}$, AcOEt e $n$-BuOH. Foram obtidas 3 frações após evaporação dos solventes: FM1 = 1,40 g; FM2 = 0,30 g e FM3 = 3,5 g. A fração FM1 foi fracionada em coluna sílica gel (70-230 mesh), eluída com gradiente de $\mathrm{CH}_{2} \mathrm{Cl}_{2} / \mathrm{MeOH}\left(\mathrm{CH}_{2} \mathrm{Cl}_{2} ; \mathrm{CH}_{2} \mathrm{Cl}_{2} /\right.$ $\mathrm{MeOH}(1,0 \rightarrow 50,0 \%) ; \mathrm{MeOH})$, fornecendo após análise por CCDA, a substância 1 (1,6 mg). A fração FM2, após cromatografia em sílica gel (70-230 mesh) utilizando como eluente $\mathrm{CH}_{2} \mathrm{Cl}_{2} / \mathrm{MeOH}$ $\left(\mathrm{CH}_{2} \mathrm{Cl}_{2} ; \mathrm{CH}_{2} \mathrm{Cl}_{2} / \mathrm{MeOH}(1,0 \rightarrow 50,0 \%) ; \mathrm{MeOH}\right)$, originou 7 frações (FM2-1 a FM2-7). Da fração FM2-3 foram obtidas as substâncias $2(10,9 \mathrm{mg})$ e 3 (3,9 mg), após cromatografia em sílica gel (70-230 mesh), eluída com gradiente de $\mathrm{CH}_{2} \mathrm{Cl}_{2} / \mathrm{MeOH}$. A fração FM2-4 foi fracionada em sílica gel (70-230 mesh) utilizando como eluente hexano/AcOEt/MeOH (hexano; hexano/AcOEt $(10,0 \rightarrow 100,0 \%)$; $\mathrm{AcOEt} / \mathrm{MeOH}(10,0 \rightarrow 100,0 \%)$, em polaridade crescente e, posteriormente, via CLAE preparativa $\left(\mathrm{MeOH} / \mathrm{CH}_{2} \mathrm{Cl}_{2} 7: 3\right)$, fornecendo a substância 4 (3,00 mg).

\section{Isolamento das substâncias de T. catigua}

O extrato metanólico do arilo dos frutos de T. catigua $(1,30 \mathrm{~g})$ foi extraído sucessivamente com $\mathrm{CH}_{2} \mathrm{Cl}_{2}$ e $\mathrm{MeOH}$. Foram obtidas 2 frações após evaporação dos solventes: FCM1 = 0,14 g; FCM2 = 0,98 g. A fração FCM1 foi submetida a vários fracionamentos em coluna de sílica gel (70-230 mesh), eluída com $\mathrm{CH}_{2} \mathrm{Cl}_{2} / \mathrm{MeOH}$ $\left(\mathrm{CH}_{2} \mathrm{Cl}_{2} ; \mathrm{CH}_{2} \mathrm{Cl}_{2} / \mathrm{MeOH}(1,0 \rightarrow 50,0 \%) ; \mathrm{MeOH}\right)$, em gradiente de polaridade crescente. Após esses fracionamentos, foi obtida a substância 5 (4,4 mg). A fração FCM2 foi fracionada em coluna de sephadex LH-20 eluída com $\mathrm{CH}_{2} \mathrm{Cl}_{2} / \mathrm{MeOH} 1: 1$, fornecendo após análise por CCDA, as frações FCM2-1 a 6. A fração FCM2-4 foi submetida à purificação por cromatografia em sílica gel (70-230 mesh) tendo como eluente o gradiente $\mathrm{CH}_{2} \mathrm{Cl}_{2} / \mathrm{MeOH}\left(\mathrm{CH}_{2} \mathrm{Cl}_{2}\right.$; $\left.\mathrm{CH}_{2} \mathrm{Cl}_{2} / \mathrm{MeOH}(1,0 \rightarrow 50,0 \%) ; \mathrm{MeOH}\right)$, fornecendo o limonoide 6 $(1,0 \mathrm{mg})$. A fração FCMC-6 foi submetida ao mesmo processo de fracionamento cromatográfico e levou ao isolamento da mistura contendo 7 e 8 (3,6 mg).

\section{RESULTADOS E DISCUSSÃO}

Ensaios biológicos realizados com extratos orgânicos de frutos de T. elegans e $T$. catigua sobre $S$. frugiperda mostraram que os extratos hexânico, metanólico e hidrometanólico de sementes de T. catigua provocaram mortalidades larvais moderadas ( $\cong 50,0 \%)$ (Tabela 1$)$. A mais alta taxa de mortalidade larval $(100,0 \%)$ foi obtida com os extratos hexânico e metanólico de frutos de T. elegans.

Para os extratos hexânico e metanólico de frutos de T. elegans foi observada incapacidade das larvas para empuparem, que pode estar relacionada com variações hormonais nos insetos afetados, pois elas não conseguiram fazer a muda. Os mesmos resultados foram obtidos para larvas tratadas com azadiractina; além disso, azadiractina tem se mostrado capaz de interferir com a ecdíse. ${ }^{14} \mathrm{Em}$ estudos anteriores, vários limonoides foram isolados dos frutos de $T$. elegans, ${ }^{13,15}$ mas suas atividades ainda não foram determinadas.

Tabela 1. Mortalidade da fase larval de S. frugiperda alimentada com dieta artificial tratada com extratos de Trichilia spp., a 1000 mg kg-1

\begin{tabular}{lccccc}
\hline & & \multicolumn{2}{c}{ Mortalidade da fase larval (\%) } \\
& & Controle & Hexânico & Metanólico & Hidrometanólico \\
\hline T. catigua & Sementes & 6,67 & 56,67 & 43,33 & 53,33 \\
& Arilo & 6,67 & $\mathrm{NT}$ & 10,00 & 13,33 \\
& Exocarpo & 6,67 & 13,33 & 13,33 & 23,33 \\
T. elegans & Frutos & 10,00 & 100,00 & 100,00 & 33,33 \\
\hline
\end{tabular}

NT - Não testada. 
Os extratos hexânico e metanólico de frutos de T. elegans apresentaram mortalidade similar ao extrato aquoso das sementes de Azadirachta indica sobre larvas de $S$. exempta, demonstrando ser um poderoso inseticida. ${ }^{14}$ Uma alta taxa de mortalidade representa uma das atividades inseticidas mais eficientes, já que atua logo nas primeiras fases da praga, diminuindo seu ataque na cultura e contribuindo para o decréscimo das populações subsequentes. Assim, o estudo deste extrato é de suma importância, seja na sua forma bruta ou visando o isolamento de seus constituintes químicos.

Ao se fracionar o extrato metanólico de frutos de T. elegans foram isoladas as cumarinas (1-3) e o limonoide (4), do extrato hexânico foram isoladas as mesmas cumarinas e os esteroides. Há na literatura relatos de atividade antialimentar de furanocumarinas sobre $S$. exigua ${ }^{16}$ e $S$. litura. ${ }^{17}$

Apesar da baixa atividade inseticida do extrato metanólico do arilo de $T$. catigua (10\%, Tabela 1) este foi fracionado e foi possível isolar os limonoides cedrelona (5), angolensato de metila (6) e a mistura epimérica de fotogedunina (7 e 8).

O estudo químico do extrato hexânico dos frutos de T. elegans resultou no isolamento e identificação das cumarinas escoporona (1), escopoletina (2), umbeliferona (3) e na identificação da mistura dos esteroides: $\beta$-sitosterol, estigmasterol, campesterol e sitostenona. As estruturas das cumarinas escoporona (1), escopoletina (2), umbeliferona (3) foram elucidadas com base nas análises dos dados de EM, RMN ${ }^{1} \mathrm{H}{ }^{13} \mathrm{C}$, além da comparação com dados publicados anteriormente. ${ }^{18} \mathrm{~A}$ mistura dos esteroides foi identificada através de análises por CG-EM, comparando-se os espectros de massas obtidos com os espectros existentes no banco de dados do equipamento, por $\mathrm{RMN}{ }^{1} \mathrm{H} \mathrm{e}{ }^{13} \mathrm{C}$ e também por comparação com dados da literatura. ${ }^{19}$ O cromatograma de íons totais da análise por CG-EM apresentou 4 picos. $\mathrm{O}$ espectro de massas dos picos correspondentes apresentou íons moleculares em $\mathrm{m} / \mathrm{z}$ 414, 412, 400 e 412, concordantes com os espectros de massas do sitosterol, estigmasterol, campesterol e sitostenona, respectivamente, sendo o esteroide sitosterol o composto majoritário desta mistura.
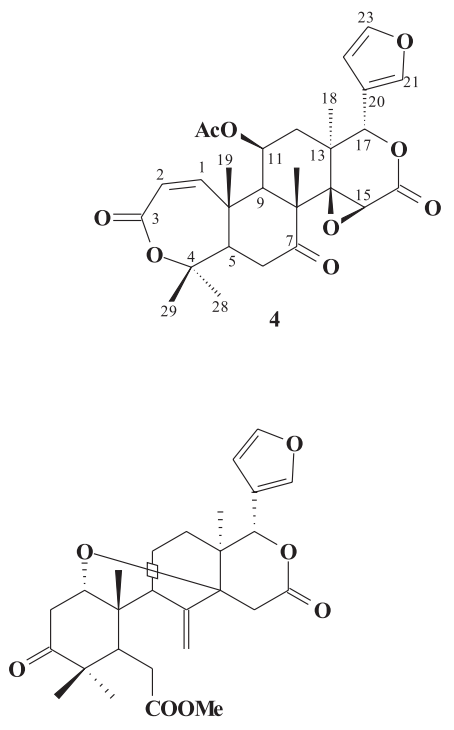

6
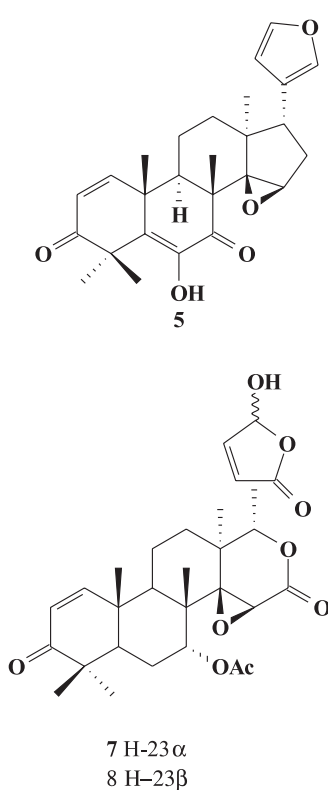

A fração de T. elegans em AcOEt, resultante da partição do extrato metanólico dos frutos forneceu o limonoide $11 \beta$-acetoxiobacunona (4) cuja estrutura foi caracterizada através de dados fornecidos pelo espectros de $\mathrm{RMN}{ }^{1} \mathrm{H}$ e ${ }^{13} \mathrm{C} 1 \mathrm{D}$ e 2D (HSQC, HMBC, Tabela 2), envolvendo comparações com dados descritos na literatura ${ }^{13,20}$ para compostos com estruturas semelhantes ao limonoide (4). Esta é a primeira vez que os dados de $\mathrm{RMN}{ }^{1} \mathrm{H}$ e ${ }^{13} \mathrm{C}$ do limonoide $11 \beta$-acetoxiobacunona (4) estão sendo apresentados. No espectro de $\mathrm{RMN}{ }^{1} \mathrm{H}$ da substância 4 foram observados sinais em $\delta 7,40-7,42(\mathrm{~m}, \mathrm{H}-21$ e H-23) e $\delta 6,35$ (s, H-22) ppm característicos do anel furano. As atribuições dos C-20 $(\delta 119,5)$, C-21 $(\delta 141,1)$, C-22 $(\delta 109,6)$ e C-23 $(\delta 143,3)$ do anel furano foram realizadas através de $\mathrm{RMN}{ }^{13} \mathrm{C}$ e confirmadas através do mapa de correlações HSQC e HMBC. O espectro de RMN ${ }^{1} \mathrm{H}$ da substância $\mathbf{4}$ mostrou cinco singletos muito intensos com integração para três hidrogênios em: $\delta 1,08\left(\mathrm{CH}_{3}-18\right), 1,45$ $\left(\mathrm{CH}_{3}-28\right), 1,50\left(\mathrm{CH}_{3}-30\right), 1,53\left(\mathrm{CH}_{3}-29\right), 1,60\left(\mathrm{CH}_{3}-19\right) \mathrm{ppm}$ referentes a grupos metila ligados a carbono $\mathrm{sp}^{3}$. Em $\delta 3,74$ e $\delta$ $5,45 \mathrm{ppm}$ observou-se a presença de dois sinais característicos do anel D $\delta$-lactônico com epóxido entre C-14 e C-15, referente aos hidrogênios ligados aos C-15 e C-17, respectivamente. A presença de uma dupla ligação conjugada a um carbono carbonílico no anel A, foi determinada pela presença de dois dubletos em $\delta$ $6,01(\mathrm{H}-2, \mathrm{~J}=12,1 \mathrm{~Hz})$ e $\delta 6,55(\mathrm{H}-1, \mathrm{~J}=12,1 \mathrm{~Hz})$ atribuídos aos hidrogênios olefínicos e ao sinal em $\delta 166,4$ referente ao carbono carbonílico em C-3. O mapa de correlações HMBC permitiu confirmar a formação de um anel A $\varepsilon$-lactônico $\alpha$ - $\beta$ insaturado, pois se observaram as correlações dos átomos de hidrogênio em $\delta 1,45\left(\mathrm{~s}, \mathrm{CH}_{3}-28\right)$ e $\delta 1,53\left(\mathrm{~s}, \mathrm{CH}_{3}-29\right)$ com os átomos de $\mathrm{C}-4(\delta$

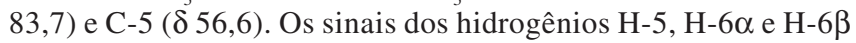
foram atribuídos através das constantes de acoplamento observadas e do mapa de correlações HMBC e HSQC. O H- $6 \alpha$ apresenta-se como tripleto largo $(3,01, \mathrm{~J}=14,2 \mathrm{~Hz})$ devido ao acoplamento geminal com o hidrogênio H-6 $\beta$ e pseudo diaxial com H-5 (dd, 2,56, J = 14,2 e 4,6 Hz).

Através de partições do extrato metanólico do arilo dos frutos de T. catigua, seguidas de fracionamentos cromatográficos, foram isolados os limonoides 5-8. As estruturas dos limonoides cedrelona (5), ${ }^{21}$ angolensato de metila $(\mathbf{6})^{22,23}$ e mistura epimérica de fotogedunina (7 e 8) ${ }^{24}$ foram elucidadas com base nas análises dos dados de RMN ${ }^{1} \mathrm{H}$ e ${ }^{13} \mathrm{C} 1 \mathrm{D}$ e $2 \mathrm{D}$ (Cosy, HSQC, HMBC), além de comparações com dados publicados anteriormente.

Todos os limonoides isolados do extrato metanólico do arilo de T. catigua apresentam atividade inseticida relevante, no entanto, foi observada uma baixa mortalidade larval deste extrato (10\%). Aparentemente esta baixa atividade está relacionada ao fato de se ter somente $1,46 \%$ de limonoides no extrato. Por outro lado, tem-se $9,80 \%$ de cedrelona (5) no extrato hexânico do arilo de T. catigua. Infelizmente, toda a massa deste extrato foi utilizada para o isolamento da cedrelona (5), deste modo não pôde ser ensaiado.

Yajima et al. ${ }^{17}$ verificaram que a cumarina umbeliferona (3) apresentou atividade antialimentar sobre Spodoptera litura L. (Lepdoptera: Noctuidae) de 45 e $77 \%$ a uma concentração de 1000 e 100 ppm, respectivamente. Já o limonoide angolensato de metila (6) mostrou atividade antialimentar no método de disco de escolha convencional no terceiro instar larval de Spodoptera littoralis (Boisd.) (Lepdoptera: Noctuidae) a $500 \mu \mathrm{g} / \mathrm{mL} .{ }^{25}$ A mistura epimérica de fotogedunina (7 e 8) apresentou mortalidade larval de $95,83 \%$ a 25,0 ppm, ao ser incorporada à dieta artificial para Spodoptera frugiperda (Lepdoptera: Noctuidae). ${ }^{26}$

Vários são os trabalhos descritos na literatura em que se demonstram as atividades inseticida e antialimentar do limonoide cedrelona (5) sobre diversas espécies de lepdópteras. ${ }^{27}$

\section{AGRADECIMENTOS}

Ao CNPq, Capes e FAPESP pelas bolsas e apoios financeiros. 
Tabela 2. Dados de $\mathrm{RMN}{ }^{1} \mathrm{H}(400 \mathrm{MHz})$ e ${ }^{13} \mathrm{C}(100 \mathrm{MHz})$ de 4 $\left(\mathrm{CDCl}_{3}\right)^{*}$

\begin{tabular}{|c|c|c|c|}
\hline $\mathrm{C} / \mathrm{H}$ & $\delta_{\mathrm{H}}$ & $\delta_{\mathrm{C}}$ & HMBC \\
\hline 1 & $6,55(\mathrm{dl}, J=12,1 \mathrm{~Hz})$ & 153,3 & H-19 \\
\hline 2 & $6,01(\mathrm{dl}, J=12,1 \mathrm{~Hz})$ & 122,4 & \\
\hline 3 & & 166,4 & $\mathrm{H}-1$ \\
\hline 4 & & 83,7 & H-28, H-29 \\
\hline 5 & $2,56(\mathrm{dd}, J=14,2$ e $4,6 \mathrm{~Hz})$ & 56,6 & 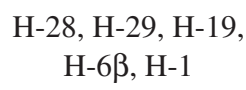 \\
\hline $6 \alpha$ & $2,44(\mathrm{dd}, J=14,2$ e $4,6 \mathrm{~Hz})$ & 38,9 & \\
\hline $6 \beta$ & $3,01(\mathrm{tl}, J=14,2 \mathrm{~Hz})$ & & \\
\hline 7 & & 205,8 & \\
\hline 8 & & 51,8 & $\mathrm{H}-30$ \\
\hline 9 & $2,15(\mathrm{sl})$ & 49,5 & H-30, H-19, H-1 \\
\hline 10 & & 43,8 & H-19, Н-9 \\
\hline 11 & $5,71(\mathrm{dd}, J=6,1$ e $2,2 \mathrm{~Hz})$ & 68,3 & \\
\hline 12 & $1,82(\mathrm{~d}, J=6,1 \mathrm{~Hz})$ & 40,8 & $\mathrm{H}-18$ \\
\hline 13 & & 36,3 & H-18 \\
\hline 14 & & 63,9 & H-18, H-30 \\
\hline 15 & $3,74(\mathrm{~s})$ & 53,55 & \\
\hline 16 & & 166,2 & $\mathrm{H}-15$ \\
\hline 17 & $5,45(\mathrm{~s})$ & 78,0 & H-18, H-12 \\
\hline 18 & $1,08(\mathrm{~s})$ & 20,3 & $\mathrm{H}-17$ \\
\hline 19 & $1,60(\mathrm{~s})$ & 17,9 & $\mathrm{H}-9$ \\
\hline 20 & & 119,5 & $\mathrm{H}-17$ \\
\hline 21 & $7,40-7,42 \mathrm{~m}$ & 141,1 & $\mathrm{H}-17$ \\
\hline 22 & $6,35(\mathrm{~s})$ & 109,6 & $\mathrm{H}-17$ \\
\hline 23 & $7,40-7,42 \mathrm{~m}$ & 143,3 & \\
\hline 28 & $1,45(\mathrm{~s})$ & 31,8 & H-29 \\
\hline 29 & $1,53(\mathrm{~s})$ & 26,3 & H-28 \\
\hline 30 & $1,50(\mathrm{~s})$ & 19,4 & H-9 \\
\hline \multirow[t]{2}{*}{ Oac } & $2,19(\mathrm{~s})$ & 21,4 & \\
\hline & & 169,9 & \\
\hline
\end{tabular}

* Espectros de $2 \mathrm{D}{ }^{1} \mathrm{H}-{ }^{1} \mathrm{H}-\mathrm{Cosy}$ e ${ }^{1} \mathrm{H}-{ }^{13} \mathrm{C}-\mathrm{HSQC}$ também foram utilizados na atribuição dos deslocamentos químicos.

\section{REFERÊNCIAS}

1. Klocke, J. A. Em Allelochemicals: Role in agriculture and forestry; Waller, G. R., ed.; ACS Symp. Ser. 330, Amer. Chem. Soc.: Washington, DC, 1987, p. 396-415.

2. Martinez, S. S.; O Nim-Azadirachta indica: Natureza, usos múltiplos, produção, Instituto Agronômico do Paraná, Londrina, 2002.

3. Bogorni, P. C.; Vendramim, J. D.; Neotrop. Entomol. 2003, 32, 665.

4. Nakatani, M.; Iwashita, T.; Naoki, H.; Hase, T.; Phytochemistry 1985, 24, 195; Nakatani, M.; James, J. C.; Nakanishi, K.; J. Am. Chem. Soc. 1981, 103, 1228.
5. Ramírez, M. C.; Toscano, R. A.; Arnason, J.; Omar, S.; Cerda-GarcíaRojas, C. M.; Mata, R.; Tetrahedron 2000, 56, 5085.

6. Mikolajczak, K. L.; Reed, D. K.; J. Chem. Ecol. 1987, 13, 99.

7. Mikolajczak, K. L.; Zilkowski, B. W.; Bartelt, R. J.; J. Chem. Ecol. 1989, 15, 121; Rodríguez, H. C.; Vendramim, J. D.; Man. Integ. Pragas 1996, 42, 14; Rodríguez, H. C.; Vendramim, J. D.; Rev. Agric. 1997, 72, 305; Roel, A. R.; Vendramim, J. D.; Frighetto, R. T. S.; Frighetto, N.; Bragantia 2000, 59, 53; Wheeler, D. A.; Isman, M. B.; Sanchez-Vindas, P. E.; Arnason, J. T.; Biochem. Syst. Ecol. 2001, 29, 347; Wheeler, D. A.; Isman, M. B.; Entomol. Exp. Appl. 2001, 98, 9; Bogorni, P. C.; Vendramim, J. D.; Neotrop. Entomol. 2005, 34, 311.

8. Matos, A. P.; Nebo, L.; Batista-Pereira, L. G.; Vieira, P. C.; Fernandes, J. B.; Da Silva, M. F. G. F.; Rodriguez, R. R.; Bioassay 2006, 1/X, 7.

9. Kubo, I.; Klocke, J. A.; Experientia 1982, 64, 281.

10. Simmonds, M. S. J.; Stevenson, P. C.; Porter, E. A.; Veitch, N. C.; J. Nat. Prod. 2001, 64, 1117.

11. Rodríguez, B.; Caballero, C.; Ortego, F.; Castañera, P.; J. Nat. Prod. 2003, 66, 452.

12. Pott, A.; Pott, V.; Plantas do Pantanal, Empresa Brasileira Agropecuária: Brasília, 1994, p. 204.

13. Garcez, F. R. ; Garcez, W. S. ; Tsutsumi, M. T. ; Roque, N. F.; Phytochemistry 1997, 45, 141; Garcez, F. R.; Garcez, W. S.; Roque, N. F.; Castellano, E. E.; Zuckerman-Schpector, J.; Phytochemistry 2000, 55, 733.

14. Tanzubil, P. B.; McCaferry, A. R.; Crop Prot. 1990, 9, 383.

15. Garcez, F. R.; Garcez, W. S.; Tsutsumi, M. T.; Roque, N. F.; Phytochemistry 1997, 45, 141

16. Berdegué, M.; White, K. K.; Trumble, J. T.; Environ. Entomol. 1997, 26, 912.

17. Yajima, T.; Munakara, K.; Agric. Biol. Chem. 1979, 43, 1701.

18. Müller, A. H.; Degáspari, L. R. O.; Vieira, P. C.; Silva, M. F. G. F. da; Fernandes, J. B.; Pirani, J. R.; Phytochemistry 1993, 34, 585.

19. Müller, A. H.; Vieira, P. C.; Silva, M. F. G. F. da; Fernandes, J. B.; Phytochemistry 1995, 40, 1797; Kong, L. Y.; Min, Z. D.; Li, X.; Zhu, T. R.; Phytochemistry 1996, 41, 1423; Goulart, M. O. I.; Sant'ana, A. E. G.; Lima, R. A.; Cavalcante, S. H.; Quim. Nova 1993, 16, 95; Sakakibara, J.; Kaya, T.; Fukuda, H.; Ohki, T.; Phytochemistry 1983, 22, 2553.

20. Mitsuo, K.; Maejima, M.; Fukaya, Y. H.; Takeya, K.; Phytochemistry 2004, 65, 3075 .

21. Luo, X.; Wu, S.; Ma, Y.; Wu, D.; J. Nat. Prod. 2000, 63, 947.

22. Paula, J. R. de; Vieira, I. J. C.; Silva, M. F. G. F. da; Rodrigues, F. E.; Fernandes, J. B.; Vieira, P. C.; Pinheiro, A. L.; Vilela, E. F.; Phytochemistry 1997, 44, 1449.

23. Kadota, S.; Marpaung, L.; Kikuchi, T.; Ekimoto, H.; Chem. Pharm. Bull. 1990, 38, 639.

24. Céspedes, C. L.; Calderón, J. S.; King-Diaz, B.; Lotina-Hennsen, B.; J. Agric. Food. Chem. 1998, 46, 2810.

25. Abdelgaleil, S. A. M.; Nakatani, M.; J. Appl. Entomol. 2003, 127, 236.

26. Céspedes, C. L.; Calderón, J. S.; Lina, L.; Aranda, E.; J. Agric. Food Chem. 2000, 48, 1903.

27. Suresh, G.; Gopalakrishnan, G.; Wesley, S. D.; Singh, N. D. P.; Malathi, R.; Rajan, S. S.; J. Agric. Food Chem. 2002, 50, 4484; Koul, O.; Isman, M. B.; Entomol. Exp. Appl. 1992, 64, 281; Arnason, J. T.; Philogéne, B. J. R.; Donskov, N.; Kubo, I.; Entomol. Exp. Appl. 1987, 43, 221; Kubo, I.; Klocke, J. A. Em Natural Resistence of Plants to Pests; Green, M. B.; P. A. Hedin, P. A., eds.; ACS Symp. Ser. 296, Amer. Chem. Soc.: Washington, DC, 1986, p. 206-219. 